\title{
Plasma Cells Leukemia Masquerading as Lymphocyte-Monocyte Peak on Automated Cell Analyzer
}

\author{
Dr. Akshita Rattan ${ }^{1 *}$, Dr. Anita Tahlan ${ }^{2}$, Dr. Swathi C Prabhu², Dr. Sanjay D'Cruz ${ }^{3}$ \\ ${ }^{1}$ Junior Resident, Department of Pathology, Government Medical College Sector-32, Chandigarh, India \\ ${ }^{2}$ Department of Pathology, Government Medical College Sector-32, Chandigarh, India \\ ${ }^{3}$ Department of Dermatology, Government Medical College Sector-32, Chandigarh, India
}

\begin{tabular}{l|l}
\hline DOI: $10.36348 /$ sjpm.2021.v06i02.006 & | Received: 03.02.2021 | Accepted: 14.02 .2021 | Published: 16.02 .2021 \\
*Corresponding author: Dr. Akshita Rattan &
\end{tabular}

\section{Abstract}

Background: Peripheral blood plasmacytosis can be seen in plasma cell leukemia (PCL) or plasma cell myeloma (PCM). As plasma cells show dysplasia or lymphocytoid morphology, they masquerade as monocytes or high fluorescent lymphocytes (HFL) on automated cell analyzer. The early suspicion and detection are of clinical importance for diagnostic and prognostic reasons. Methods: Automated cell analyzer was used for routine CBC examination. Peripheral blood smear examination was performed for enumeration and characterization of cells in peripheral blood followed by bone marrow examination and ancillary techniques. Conclusion: Lymphocyte-Monocyte peak with increased high HFL count on $\mathrm{CBC}$ should prompt a smear examination of the peripheral blood for circulating plasma cells and immunohistochemistry markers like CD38, CD138, CD56 should be used to establish the clonality in cells.

Keywords: plasma cell leukemia (PCL), high fluorescent lymphocytes (HFL), Peripheral blood plasmacytosis.

Copyright (C) 2021 The Author(s): This is an open-access article distributed under the terms of the Creative Commons Attribution 4.0 International License (CC BY-NC 4.0) which permits unrestricted use, distribution, and reproduction in any medium for non-commercial use provided the original author and source are credited.

\section{INTRODUCTION}

PCL is a rare disease and is the most aggressive entity of the plasma cell neoplasms [1, 2]. It accounts for $2-3 \%$ of all plasma cell dyscrasias and usually presents after the fifth decade of life [3, 4]. PCL may appear either de novo (primary form) or may occur in patients with long standing cases of multiple myeloma (secondary form). The diagnostic definition of PCL is based on Kyle's criteria. It is characterized by the presence of at least $20 \%$ of plasma cells in circulation and at least of, total plasma cell count of $2 \times 10^{9} / \mathrm{L}$ in peripheral blood. The plasma cells may resemble plasmacytoid lymphocytes [1, 3, 5]. Plasma cell leukemia is derived from terminally differentiated $\mathrm{B}$ cells as a response to humoral immunity [3]. As normally plasma cells reside in blood circulation for only a small period, the large numbers are considered to be pathological. Increased numbers of plasma cells are in conditions like infections, auto-immune disease and hematological malignancy such as plasma cell myeloma and plasma cell leukemia. Careful examination of CBC scatterplot is very essential for initial suspicion in a clinical setting of myeloma, as it influences early diagnosis and treatment. Thus, the ability of automated cell analyzers to differentiate plasma cells is of utmost importance [6].

Here we present a case of where the plasma cells are masquerading in the lymphocyte monocyte region as flagged by the analyzer prompting us for thorough morphological evaluation of peripheral smear and accurately diagnosing the case as plasma cell leukemia.

\section{CASE REPORT}

A 67 years old male patient, a known case of Type 2 diabetes mellitus, chronic kidney disease and diabetic nephropathy, presented with complaints of decreased urine output and pedal edema. Routine biochemical tests showed hypercalcemia $(14.5 \mathrm{mg} / \mathrm{dl})$, hyperuricemia $(200 \mathrm{mg} / \mathrm{dl})$, renal insufficiency (creatinine levels $5.5 \mathrm{mg} / \mathrm{dl}$ ). CBC performed on a Sysmex XN 1000 hematology analyzer (Kobe, Japan) showed anemia (hemoglobin $7.4 \mathrm{~g} / \mathrm{dl}$ ), Total leukocyte count $\left(25.85 \times 10^{3} / \mu \mathrm{L}\right)$ with differential of neutrophils (11.3\%), lymphocytes $(47.9 \%)$ and absolute monocytosis (39.8\% of leukocytes), HFLC count of $0.06 \times 10^{3} / \mu \mathrm{L}(0.2 \%)$ and platelet count $\left(24 \times 10^{3} / \mu \mathrm{L}\right)$. The analyzer flagged abnormal WBC scattergram for 
Akshita Rattan et al; Saudi J Pathol Microbiol, Feb, 2021; 6(2): 76-79

lymphocytosis, monocytosis, blasts/atypical lymphocytes. The large number of cells in the monocytic and blastic/atypical lymphocytes region of differential scatterplot possibly showed abnormal plasma cells with increased fluorescence as shown in the Figure-1. The abnormal scatterplot made us look into the peripheral blood smear which showed $15 \%$ neutrophils, $19 \%$ lymphocytes, $60 \%$ plasma cells including plasmacytoid lymphocytes which was flagged by analyzer as monocytes. However, smear showed only $6 \%$ monocytes. The Bone marrow aspiration revealed dry tap. Counts were done on imprint smear which showed $79 \%$ plasma cells, including binucleated and multinucleated plasma cells and $1 \%$ plasma blasts. On further evaluating, $\mathrm{M}$ band was noted on serum and urine electrophoresis. Biopsy showed sheets of plasma cells which was positive for CD 138. Congo red was done to detect amyloid and was negative.

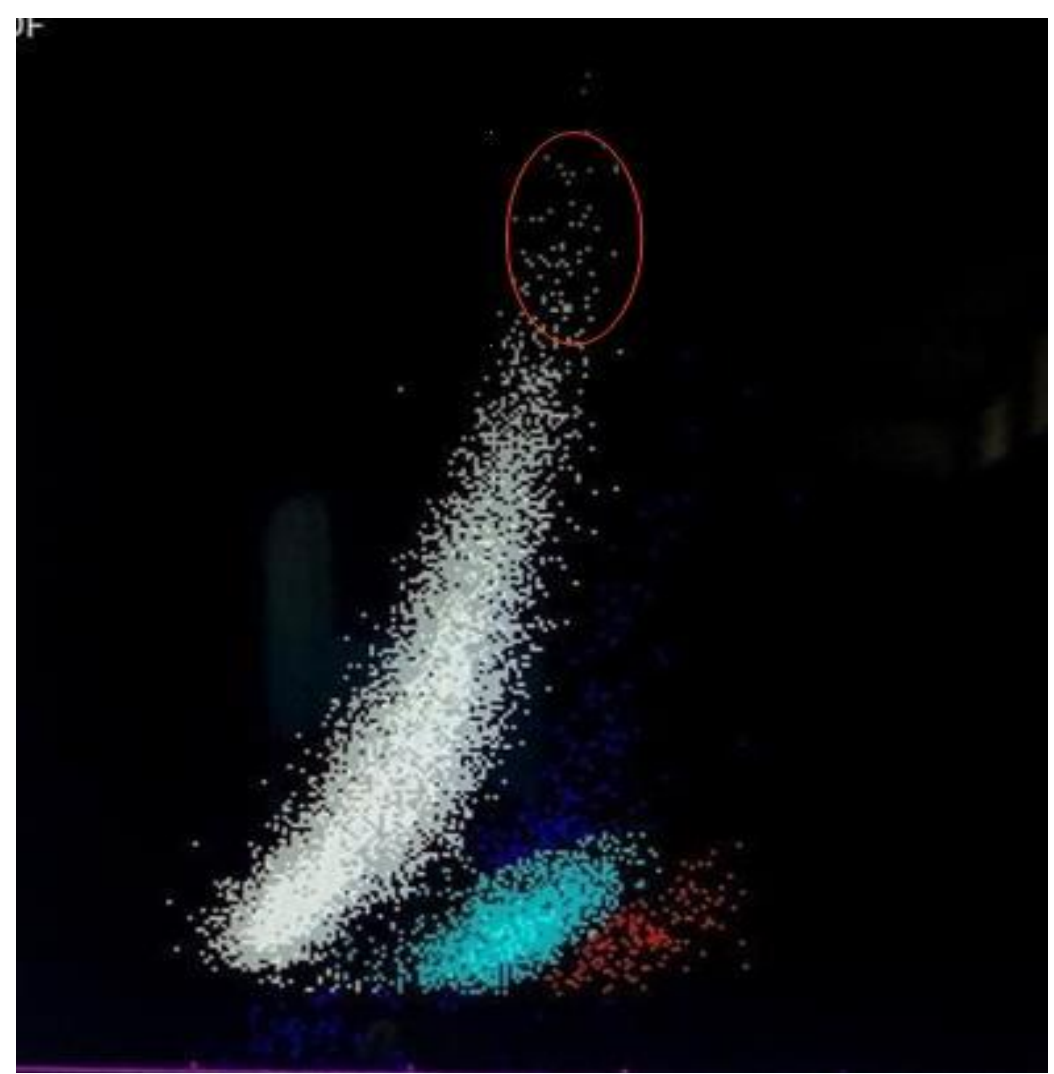

Fig-1: CBC leukocyte differential scatter plot showing a united population in the lymphocytic and monocytic region, and the majority of cells with increased fluorescence lying in the lymphocyte-monocytic and blastic/atypical lymphocytes region. The circle possibly represents abnormal cells with high fluorescence

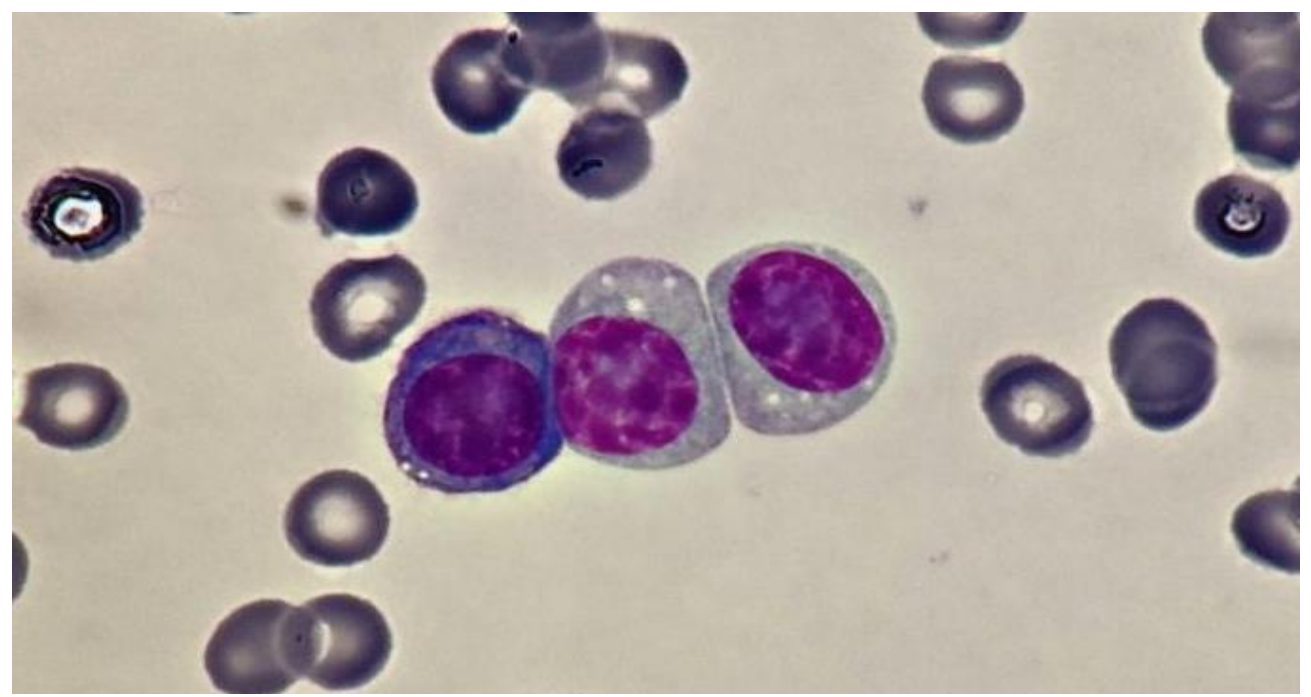

Fig-2: Peripheral smear with plasma cells (100X, Leishman stain) displaying mature chromatin, eccentric placed nucleus with basophilic cytoplasm 


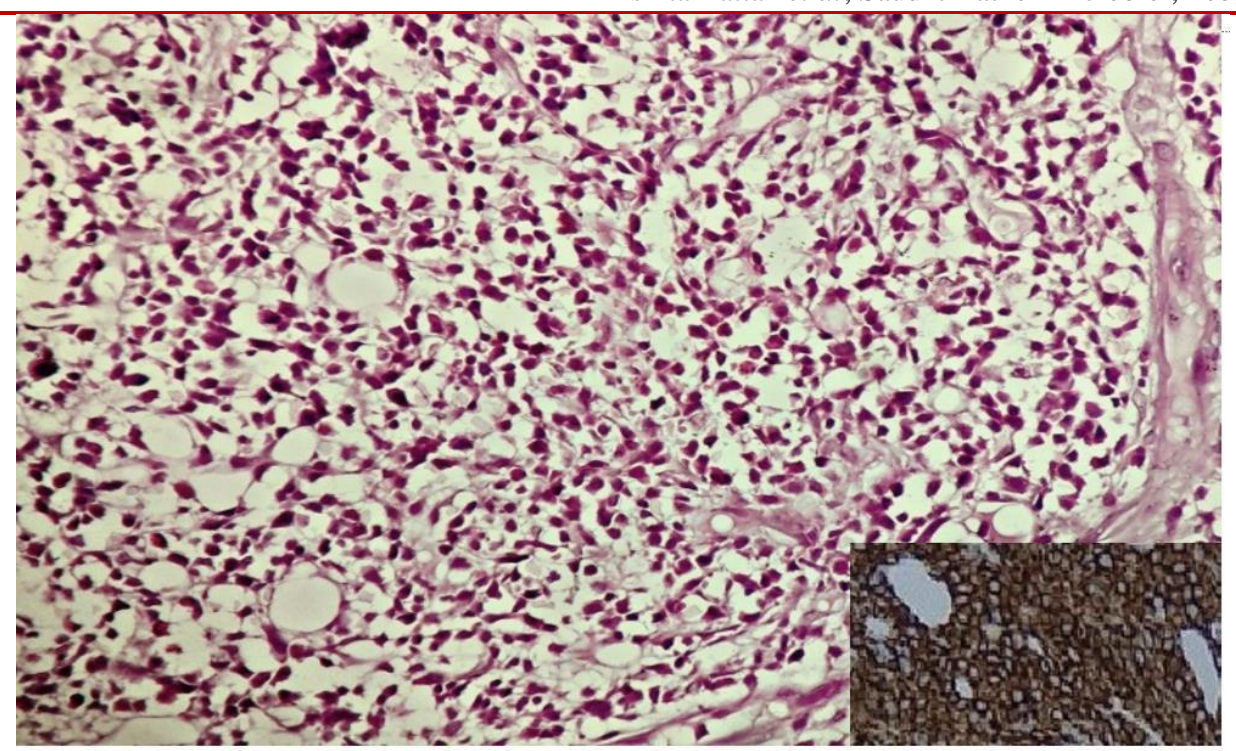

Fig-3: Bone marrow biopsy shows diffuse infiltration by plasma cells (H\&E, 40x) and inset shows CD 138 IHC which is diffusely positive in plasma cells

\section{DISCUSSION}

In this report we present a case of plasma cell leukemia with high HFL count due to increased plasma cells in circulation. The automated cell analyzer the Sysmex XN-1000 hematology analyzer which is a sixpart differential analyzer used in this study uses optical light scatter principle and hydrodynamic focusing detection to calculate and observe the size of cells using a laser beam. The electrical signals generated is proportional to the number of cells that go through the sensing zone [7] and, the combination of side scatter (inner complexity of the cell), forward scatter (size of the cell) and fluorescence intensity (RNA/DNA content) of nucleated cells distinguishes the leukocyte in to subpopulations of eosinophils, basophils, neutrophils, lymphocytes, monocytes, and immature granulocyte can be calculated on it [6].

Sysmex XN-1000 is also able to perform high fluorescent lymphocytes (HFL) count. HFLs bind more nucleic acid fluorescent dyes leading to high fluorescence activity in atypical lymphocyte zones on scatterplot; and for the same reason the raised HFL count is observed in plasma cell leukemia [7, 8]. Since plasma cells are rich in nucleic acids, these are counted under a distinct population of cells called high fluorescent lymphocytes (HFL) [6].

The HFL count has been suggested for screening elevated plasma cells. As no other hematology parameter is suggested for flagging the possible presence of circulating plasma cells, the absolute lymphocytosis, monocytosis observed on a digital differential analyzer, raised HFL count and CBC scatterplots should be the main triggers to look for circulating plasma cells [1]. Normal range of HFLC is 0 to $1 \%$. However, HFL count measured by the analyzer does overestimate the plasma cell count. This is because the HFL population measured by analyzer includes not only plasma cells but also other lymphocytes with high RNA content. Other causes of high HFL count but low plasma cell count includes patients with reactive granulocytosis, generally seen in response to infection [6]. In addition, false negative results may appear in the specimens, with little tumor cells [8]. Thus, prompt and careful examination of peripheral smear has to be done in these cases. Studies done by Gounari et al., Fernandez et al., and Nowakowski et al., also reinforce the significance of careful examination of HFLC and monocyte count on the CBC $[1,9,10]$.

The accurate diagnosis of plasma cell leukemia still lies in the precise counting of circulating plasma cells on peripheral smears though atypical morphology may lead to misrecognition and miscounting of cells leading to low reproducibility. Though diagnosing PCL using flow cytometric detection of abnormal circulating plasma cells is not established, it is of prognostic significance in cases of plasma cell myeloma [1].

\section{CONCLUSION}

In conclusion we suggest that lymphocytosis, monocytosis and increased high HFL count on the scatter plot on CBC should prompt a smear examination of the peripheral blood for circulating plasma cells and immunohistochemistry markers like CD38, CD138, CD56 should be used to establish the clonality in cells.

Conflict of Interest: The authors declare that they have no conflict of interest.

\section{REFERENCES}

1. Gounari, E., Tsavdaridou, V., Koletsa, T., Nikolaidou, A., Kaiafa, G., Papaioannou, M., ... \& Skoura, L. (2016). Utility of hematology analyzer 
Akshita Rattan et al; Saudi J Pathol Microbiol, Feb, 2021; 6(2): 76-79

and flow cytometry in timely and correct detection of circulating plasma cells: Report of three cases. Cytometry Part B: Clinical Cytometry, 90(6), 531-537.

2. Wöhrer, S., Ackermann, J., Baldia, C., Seidl, S., Raderer, M., Simonitsch, I., \& Drach, J. (2004). Effective treatment of primary plasma cell leukemia with thalidomide and dexamethasone-a case report. The hematology journal: the official journal of the European Haematology Association, 5(4), 361-363.

3. Jameel A. Plasma Cell Leukemia: Case Report of a Rare and Aggressive Variant of Multiple Myeloma. :2.

4. Elsabah, H., Soliman, D. S., Ibrahim, F., AlSabbagh, A., Yassin, M., Moustafa, A., ... \& ElOmri, H. M. (2020). Plasma Cell Myeloma with an Aggressive Clinical Course and Anaplastic Morphology in a 22-Year-Old Patient: A Case Report and Review of Literature. The American journal of case reports, 21, e920489-1.

5. Gundesen, M. T., Lund, T., Moeller, H. E., \& Abildgaard, N. (2019). Plasma cell leukemia: definition, presentation, and treatment. Current oncology reports, $21(1), 8$.

6. van Mirre, E., Vrielink, G. J., Tjon-a-Tsoi, N., Hendriks, H., de Kieviet, W., \& ten Boekel, E. (2011). Sensitivity and specificity of the high fluorescent lymphocyte count-gate on the Sysmex XE-5000 hematology analyzer for detection of peripheral plasma cells. Clinical Chemistry and Laboratory Medicine (CCLM), 49(4), 685-688.

7. Raharjo, B., \& Hadi, S. (2019). High Fluorescent Lymphocyte Count Examination in Dengue Hemorrhagic Patients With Sysmex XN-1000 Hematology Analyzer. Indonesian Journal of Clinical Pathology And Medical Laboratory, 25(2), 207-210.

8. Xu, W., Yu, Q., Xie, L., Chen, B., \& Zhang, L. (2017). Evaluation of Sysmex XN-1000 hematology analyzer for cell count and screening of malignant cells of serous cavity effusion. Medicine, 96(27).

9. De Larrea, C. F., Kyle, R. A., Durie, B. G., Ludwig, H., Usmani, S., Vesole, D. H., ... \& Shah, J. J. (2013). Plasma cell leukemia: consensus statement on diagnostic requirements, response criteria and treatment recommendations by the International Myeloma Working Group. Leukemia, 27(4), 780-791.

10. Nowakowski, G. S., Witzig, T. E., Dingli, D., Tracz, M. J., Gertz, M. A., Lacy, M. Q., ... \& Rajkumar, S. V. (2005). Circulating plasma cells detected by flow cytometry as a predictor of survival in 302 patients with newly diagnosed multiple myeloma. Blood, 106(7), 2276-2279. 